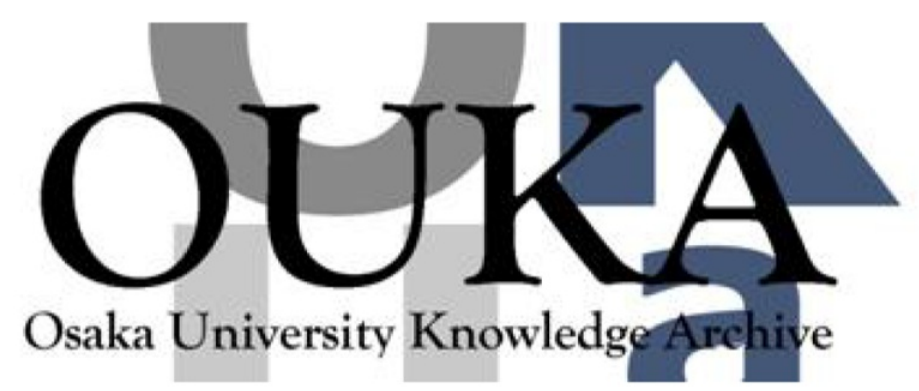

\begin{tabular}{|c|l|}
\hline Title & $\begin{array}{l}\text { Relativistic laser channeling into high-density } \\
\text { plasmas }\end{array}$ \\
\hline Author(s) & Lei, A.L.; Tanaka, K. A.; Pukhov, A. et al. \\
\hline Citation & Journal De Physique. IV : JP. 133 p. 409-p. 412 \\
\hline Issue Date & $2006-06$ \\
\hline oaire:version VoR \\
\hline URL & https://hdl. handle. net/11094/3396 \\
\hline rights & \\
\hline Note & \\
\hline
\end{tabular}

Osaka University Knowledge Archive : OUKA

https://ir. Library. osaka-u. ac. jp/

Osaka University 


\title{
Relativistic laser channeling into high-density plasmas
}

\author{
A.L. Lei ${ }^{1, *}$, K.A. Tanaka ${ }^{1,2}$, A. Pukhov ${ }^{3}$, R. Kodama ${ }^{1}$, T. Yabuuchi ${ }^{1}$, \\ K. Adumi ${ }^{1}$, R.R. Freeman ${ }^{4}$, Y. Izawa ${ }^{1}$, Y. Kitagawa ${ }^{1}$, K. Kondo ${ }^{1}$, G.R. Kumar ${ }^{5}$, \\ T. Matsuoka ${ }^{6}$, K. Mima $^{1}$, T. Norimatsu ${ }^{1}$, O. Shorokhov ${ }^{3}$, R. Snavely ${ }^{7}$ \\ and J. Zheng ${ }^{8}$
}

1 Institute of Laser Engineering, Osaka University, 2-6 Yamada-oka, Suita, Osaka 565-0871, Japan

2 Department of Electronic, Information Systems and Energy Engineering, Osaka University, 2-1 Yamada-oka, Suita, Osaka 565-0871, Japan

${ }^{3}$ Institut fur Theoretische Physik I, Heinrich-Heine-Universitat Duesseldorf, Duesseldorf 40225, Germany

${ }^{4}$ College of Mathematical and Physical Sciences, the Ohio State University, Columbus, Ohio 43210, USA

${ }^{5}$ Tata Institute of Fundamental Research, Mumbai 400005, India

6 University of Michigan, Center for Ultrafast Optical Science, Ann Arbor, MI 48109-2099, USA

${ }^{7}$ Lawrence Livermore National Laboratory, University of California, Livermore, CA 94550, USA

${ }^{8}$ Department of Modern Physics, University of Science and Technology of China, Hefei, Anhui 230026, China

\begin{abstract}
We experimentally studied relativistic laser propagation in preplasmas at the highest powers ever attempted- 0.2 to 0.4 petawatt. We demonstrated a single conical-shaped plasma channel formation extending several hundred microns in length from the under dense to over dense plasmas, indicating wholebeam self-focused laser channeling into the high-density plasma. The channel cone was reproduced by a three dimensional particle-in-cell simulation. The confirmation of the relativistic laser channeling into high-density plasmas holds the promise of fast igniting a highly compressed fuel plasma core.
\end{abstract}

\section{INTRODUCTION}

Exploration of the behavior of matter in extraordinarily large light fields is a new frontier in light-matter interaction studies. The subject continues to grow at an explosive pace, and not only new physics, but many attractive applications are beginning to emerge. For example, energetic particles, such as hot electrons, fast protons and ions, $\gamma$ rays, and neutrons etc, can be generated in these interactions. These particle sources have many potential applications such as table-top particle acceleration. A promising application of the high energy electrons and protons is the fast ignition (FI) scheme that is expected to lead to fast track realization of inertial confinement fusion [1-4]. In the FI scheme, the imploded highdensity plasma core is heated to several $\mathrm{keV}$ temperature to trigger a nuclear fusion burn wave by the hot electrons or protons generated in the relativistic laser plasma interaction. This scheme requires that an intense relativistic heating laser pulse penetrates as close as possible to the precompressed high-density core plasma. In order for the heating laser pulse to channel in the plasma, however, complex nonlinear processes in intense laser-plasma interactions, such as laser beam breakup and filament formation [5], Raman scattering [6], and propagation instabilities [7] should be carefully controlled and minimized.

*E-mail: lal@ile.osaka-u.ac.jp 
Relativistic laser channeling in underdense and overdense plasmas has been extensively explored both computationally and experimentally [5-20], where different plasma density profiles, laser powers, and timings between the laser pulses for preplasma creation and channeling have been considered. However, laser channeling in a plasma consisting of both extended underdense and overdense regions has not been yet observed. We here report experimentally and computationally successful relativistic laser channeling with whole-beam self-focusing into the high-density plasma. We demonstrate a single conical-shaped plasma channel formation extending several hundred microns in length from the under dense into over dense plasma. The conic plasma channel observed in our experiment and simulation could be generated in a highly compressed fuel plasma by the leading edge of a relativistic laser pulse, and the existing cone can in turn naturally guide the residual laser pulse to the cone end which is close to the compressed fuel core. Our work therefore holds the promise of achieving compact laser fusion energy with a natural cone, a simpler configuration than the originally proposed fast ignition scheme [1] and the improved Au cone-in-shell scheme [2-4]. Successful laser channeling into high-density plasmas also provides unique applications for material science, laboratory astrophysics, and biology, such as collimated high-density $\mathrm{MeV}$ electron beam production, the associated super-strong magnetic field for studying the phase structure and isochoric heating for an equation of state in an extreme condition with magnetic field effects.

\section{EXPERIMENTAL SETUP}

The experiment was performed on the PetaWatt (PW) laser at the Institute of Laser Engineering, Osaka University. The PW laser is a prepulse-free sub picosecond pulse $\mathrm{Nd}$ : glass laser system of 0.9 petawatt peak power. The experimental setup is shown in Fig. 1. A $0.527 \mu \mathrm{m}$ Gekko XII (GXII) laser beam with pulse duration of $1.3 \mathrm{~ns}$ full width at half maximum (FWHM) was focused through a random phase plate onto foil targets to generate preplasmas. The PW laser was focused into the plasma from the same side as the GXII beam, $0.15 \mathrm{~ns}$ after the peak of the GXII beam. The PW laser energy was $150-250 \mathrm{~J}$ in the experiment, with pulse duration of $600 \mathrm{fs}$ (FWHM) and focus size of $70 \mu \mathrm{m}$, giving peak intensities of $(0.65-1.1) \times 10^{19} \mathrm{~W} / \mathrm{cm}^{2}$. The time-integrated $\mathrm{keV} x$-ray images were obtained with a charge coupled device (CCD) backed $x$-ray pinhole camera (XPHC), placed in front of the target. Hot electron energy spectra in the 1-100 MeV range were obtained with an electron spectrometer [21], placed behind the target. The transmitted PW laser energy through the plasma was measured via the imaging system consisting of an optical diffuser placed behind the target.

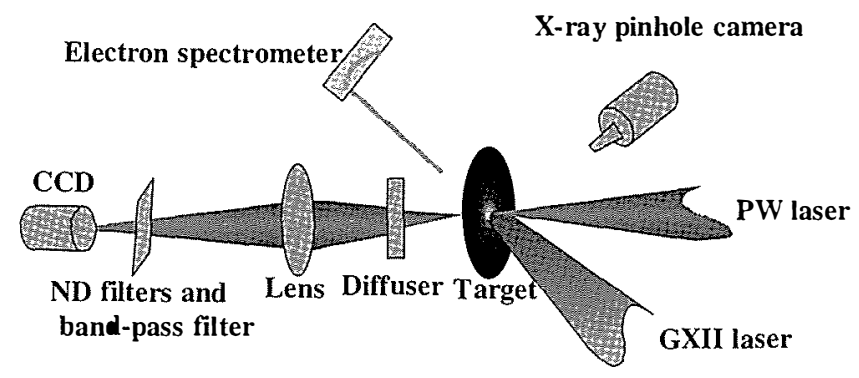

Figure 1. Experimental setup.

\section{RESULTS AND DISCUSSION}

Fig. 2 shows the raw XPHC image from 0.25 petawatt $149 \mathrm{~J}$ laser interaction with the plasma. The figure is elongated by 2.65 times in the horizontal direction for the easy recognition of detail. The PW laser focus point was set $80 \mu \mathrm{m}$ behind the target surface. The large plasma shown as the red ellipse is due to the GXII beam irradiation on the foil target. The most striking feature shown in Fig. 2 is that there is a 


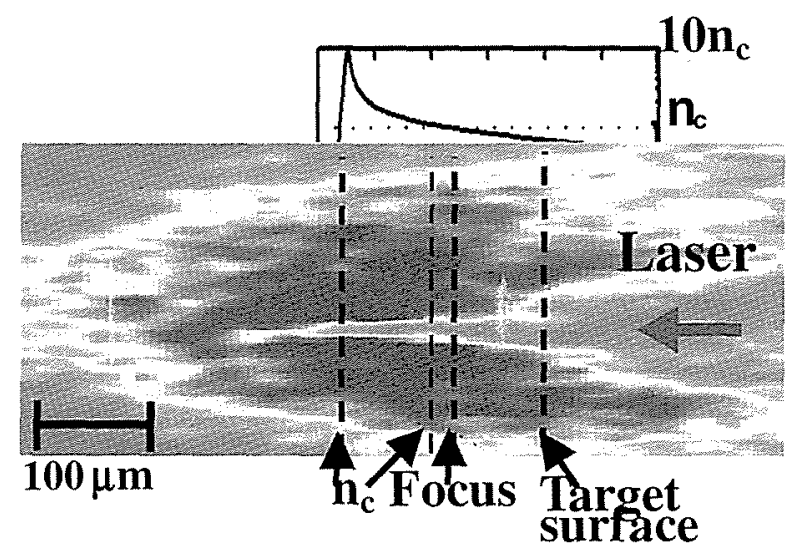

Figure 2. $x$-ray pinhole image. The red ellipse area indicates the $x$-ray emission from the preplasma while the conical shape shown in the faint $x$-ray emission region is caused by the PW laser pulse. Shown above is the simulated plasma density profile. The red color denotes relatively high $x$-ray intensity.

channel formation shown as a faint $\mathrm{x}$-ray emission region along a line in the ellipse, in contrast to the relatively strong $\mathrm{x}$-ray emission in the rest of the ellipse. The faint streak shows a conical shape, wide at the beginning, and narrow towards the end extending in more than $300 \mu \mathrm{m}$ distance. The axis of the cone is coincident with the PW laser. There was no such conical shape observed when only GXII beam irradiated the target. These two observations indicate the PW laser propagation in the plasma.

The PW laser comes from the right as marked in Fig.2a relative to the original target and the critical density surfaces. The plasma density profile was simulated by a ID hydrodynamic code ILESTA_ID [22] and was calibrated with earlier experiments $[23,24]$. The plasma had a peak density of $10 n_{c}$ with overdense region thickness of $79 \mu \mathrm{m}$. Here $n_{c}$ is the plasma critical density. The plasma density is $0.6 n_{c}$ at the original target surface, and $0.86 n_{c}$ at the PW laser focus point. One can see that the laser pulse rapidly undergoes whole-beam relativistic self-focusing after the target surface position. The self-focused laser pulse then penetrates into the plasma with density as high as $10 n_{c}$, retaining a straightforward narrow conic channel with nearly constant cross section of about $24 \mu \mathrm{m}$ ( FWHM).

The faint streak shown in Fig. 2 can be understood to be the plasma channel formed by the PW laser pulse as it propagates through the plasma. This plasma channel has less electrons and ions, and therefore emits less $\mathrm{x}$-rays than the other plasma regions, leading to the contrast of $\mathrm{x}$-ray intensity on the XPHC image shown as the faint streak.

There is difference in the values of transmitted PW laser energy through the plasma when the PW laser pulse focus point is different. We find when the laser pulse is focused at $0.86 n_{c}$ this value is estimated to be $3.8 \%$. However, only $6 \times 10^{-5}$ of transmitted PW laser energy is obtained when the laser focus point is changed to $0.6 n_{c}$, indicating that the laser channeling in the plasma is strongly dependent on its focus position.

We performed 3D VLPL particle-in-cell (PIC) simulation [25] to reproduce the channel cone structure in the over dense plasma. The simulation reveals that a conic plasma channel can be created via laser holeboring and relativistic induced transparency processes, and the collimation of associated hot electrons generated helps to stabilize the direction of the channel boring.

\section{CONCLUSION}

In conclusion, we experimentally and computationally investigate $0.2-0.4$ petawatt laser channeling in the long scale length plasmas. Laser channeling with whole-beam relativistic self-focusing in the underdense 
and overdense plasmas is demonstrated. This confirmation of the super-penetration regime will enable us to fast ignite a highly compressed fuel core.

\section{Acknowledgements}

We acknowledge all the technical support of the engineering staff at the Institute of Laser Engineering for the laser operation, target fabrication, and data acquisition. A. L. L. acknowledges the research fellowship supported by Japan Society for the Promotion of Science (JSPS).

\section{References}

[1] Tabak, M. et al., Phys. Plasmas 1 (1994), 1626-1634.

[2] Kodama, R. et al., Nature 412 (2001), 798-802.

[3] Key, M. H., Nature 412 (2001), 775-776.

[4] Kodama, R. et al., Nature 418 (2002), 933-934.

[5] Tanaka, K. A. et al., Phys. Rev. E 62 (2000), 2672-2677.

[6] Miyakoshi, T. et al., Phys. Plasmas 9 (2002), 3552-3557.

[7] Najmudin, Z. et al., Phys. Plasmas 10 (2003), 438-442.

[8] Pukhov, A. et al., Phys. Rev. Lett. 79 (1997), 2686-2689.

[9] Tatarakis, M. et al., Nature 415 (2002), 280.

[10] Banerjee, S. et al., J. opt. Soc. Am. B 20 (2003), 182-190.

[11] Borisov, A. B. et al., Phys. Rev. Lett. 68 (1992), 2309-2312.

[12] Vshivkov, V. A. et al., Phys. Plasmas 5 (1998), 2727-2741.

[13] Wilks, S. C. et al., Phys. Rev. Lett. 69 (1992), 1383-1386.

[14] Monot, P. et al., Phys. Rev. Lett. 74 (1995), 2953-2956.

[15] Borghesi, M. et al., Phys. Rev. Lett. 78 (1997), 879-882.

[16] Zepf, M. et al., Phys. Plasmas 3 (1996), 3242-3244.

[17] Fuchs, J. et al., Phys. Rev. Lett. 80 (1998), 2326-2329.

[18] Willi, O. et al., Laser and Particle Beams 19 (2001), 5-13.

[19] Kodama, R. et al., Phys. Plasmas 8 (2001), 2268-2274.

[20] Tanaka, K. A. et al., Phys. Plasmas 7 (2000), 2014-2022.

[21] Tanaka, K. A. et al., Rev. Sci. Instrum. 76 (2005), 013507.

[22] Takabe, H. et al., Phys. Fluids 31 (1988), 2884-2893.

[23] Tanaka, K. A. et al., Phys. Rev. E 60 (1999), 3283-3288.

[24] Takahashi, K. et al., Phys. Rev. Let. 84 (2000), 2405-2408.

[25] Pukhov, A., Rep. Prog. Phys. 66 (2003), 47-101. 